\title{
Sound Velocity Measurement of Shock-Compressed Quartz at Extreme Conditions
}

\author{
Liang Sun ${ }^{1}$, Huan Zhang ${ }^{1}$, Zanyang Guan ${ }^{1}$, Weiming Yang ${ }^{1}$, Youjun Zhang ${ }^{2}$, Toshimori Sekine ${ }^{3, *}$, \\ Xiaoxi Duan ${ }^{1, *}$, Zhebin Wang ${ }^{1}$ and Jiamin Yang ${ }^{1}$ \\ 1 Laser Fusion Research Center, Chinese Academy of Engineering Physics, Mianyang 621900, China; \\ sun.is.liang@gmail.com (L.S.); helenzhang1986@gmail.com (H.Z.); gzy0707@mail.ustc.edu.cn (Z.G.); \\ ywm835093137@yeah.net (W.Y.); zhebinw@vip.sina.com (Z.W.); yjm70018@sina.com (J.Y.) \\ 2 Institute of Atomic and Molecular Physics, Sichuan University, Chengdu 610065, China; \\ zhangyoujun@scu.edu.cn \\ 3 Center for High Pressure Science and Technology Advanced Research (HPSTAR), Shanghai 201203, China \\ * Correspondence: toshimori.sekine@hpstar.ac.cn (T.S.); xiaoxi_duan@163.com (X.D.)
}

check for updates

Citation: Sun, L.; Zhang, H.; Guan Z.; Yang, W.; Zhang, Y.; Sekine, T.; Duan, X.; Wang, Z.; Yang, J. Sound Velocity Measurement of

Shock-Compressed Quartz at

Extreme Conditions. Minerals 2021, 11, 1334. https://doi.org/10.3390/ $\min 11121334$

Academic Editors: Julien Siebert, Lidong Dai, Haiying Hu and Jianjun Jiang

Received: 30 October 2021

Accepted: 16 November 2021

Published: 28 November 2021

Publisher's Note: MDPI stays neutral with regard to jurisdictional claims in published maps and institutional affiliations.

Copyright: (c) 2021 by the authors. Licensee MDPI, Basel, Switzerland. This article is an open access article distributed under the terms and conditions of the Creative Commons Attribution (CC BY) license (https:/ / creativecommons.org/licenses/by/ $4.0 /)$.

\begin{abstract}
The physical properties of basic minerals such as magnesium silicates, oxides, and silica at extreme conditions, up to $1000 \mathrm{~s}$ of GPa, are crucial to understand the behaviors of magma oceans and melting in Super-Earths discovered to data. Their sound velocity at the conditions relevant to the Super-Earth's mantle is a key parameter for melting process in determining the physical and chemical evolution of planetary interiors. In this article, we used laser indirectly driven shock compression for quartz to document the sound velocity of quartz at pressures of $270 \mathrm{GPa}$ to $870 \mathrm{GPa}$ during lateral unloadings in a high-power laser facility in China. These measurements demonstrate and improve the technique proposed by Li et al. [PRL 120, 215703 (2018)] to determine the sound velocity. The results compare favorably to the SESAME EOS table and previous data. The Grüneisen parameter at extreme conditions was also calculated from sound velocity data. The data presented in our experiment also provide new information on sound velocity to support the dissociation and metallization for liquid quartz at extreme conditions.
\end{abstract}

Keywords: laser shock compression; sound velocity; high-pressure; quartz; Grüneisen parameter; super-earth

\section{Introduction}

Thousands of exoplanets such as CoRoT or Kepler outside our Solar system were discovered, raising fundamental questions about unique planetary formation mechanism [1-3] and their corresponding interior structures and dynamics [4]. Among these numerous planets, a significant proportion are to a large degree made of silica and silicate, although not all are entirely rocky [5-7]. Among these constituents, silica $\left(\mathrm{SiO}_{2}\right)$ play an important role in stable magma oceans, which are relevant to dynamo and magnetic field generation and heat transfer as well as the habitability for exoplanets [8,9]. Characterizing the equation of state (EoS), phase diagram, metallization, and dissociation process of silica at extreme conditions in exoplanets is of evident important for understanding the properties of magma oceans [10] and melting in Super-Earth interior [11].

The high-pressure behavior of silica was the subject of extensive experimental studies [12-16]. A number of stable crystalline structure and metastable forms of $\mathrm{SiO}_{2}$ were observed experimentally or predicted theoretically $[17,18]$, but pressure is limited in most studies to below $200 \mathrm{GPa}[19,20]$. The properties of silica in Super-Earth interiors are still poorly constrained regarding the extreme conditions in pressure and temperature.

Laboratory laser driven shock loading presents a unique capability to yield $\mathrm{TPa}$ pressure-temperature states comparable to planetary interior [1,21]. As an impedancematching standard material, the principle Hugoniot [22-25] and release isentrope [26,27] 
of $\alpha$-quartz were studied extensively in the fluid region. Shock-induced vaporization of quartz occurs at much lower pressures than previous estimates because of the entropy on the Hugoniot [28]. Melting temperatures up to $8300 \mathrm{~K}$ above $500 \mathrm{GPa}$ for fused silica, $\alpha$-quartz and stishovite were reported in laser-driven shock experiments [29]. For further increases in pressure along the Hugoniot, silica transitions form a bonded molecular liquid to a dissociated atomic fluid provided by specific heat $c_{v}$ information [24], and the evolution of the electronic structure [30]. The metallization and dissociation in silica influence strongly the equation of state and evolution of the associated transport properties.

Sound velocity describes the off-Hugoniot properties of a material and offers constraints on presence of chemical bonds based on a thermodynamically consistent analysis. The experimental determination of the speed of sound behind a shock front is of great interest for studying phase transition and geophysical problems [31-34]. The Grüneisen coefficient could be also determined by the sound velocity along a known Hugoniot curve. The study of sound velocity for silica in high pressure by dynamic shock experiments could provide further information on dissociation and metallization and increase our confidence for our theoretical models for Super-Earth interior condition.

In this article, we present high-pressure sound velocity and Grüneisen coefficient by laser shock compression experiment for $\alpha$-quartz. These experiments revisited and provided absolute measurement of sound velocity in $\alpha$-quartz from 200 to $900 \mathrm{GPa}$ by improving the previous technique proposed by Li et al. [35]. The experimental results are compared to SESAME EoS table, in which there are various theoretical and experimental results by different platforms for various silica polymorphs.

\section{Methods}

\subsection{Sound Velocity Method by Edge Rarefaction}

Methods for measuring the speed of sound behind a shock front were developed by $\mathrm{Al}^{\prime}$ tshuler et al. [36,37]. Method of lateral unloading is one of them, in which disturbances from the unloading overtake the shock front and weaken the shock. The front velocity in the weakened outer section of the front surface decreases and the shock velocity image on the streak curves, while the central part not yet disturbed remains planar. The trace of the bent location provides the speed of sound and the shock velocity as a function of transit time. The sound velocity $c_{S}$ can be obtained by

$$
c_{s}=u_{s} \times\left(\tan ^{2} \alpha+\left(\frac{u_{s}-u_{p}}{u_{s}}\right)^{2}\right)^{1 / 2},
$$

where $u_{s}$ is shock velocity and $\alpha$ is unloading angle between the shock direction and the tangent of bending trance. $u_{p}$ is the particle velocity behind shock. But the location where shock front bends and sound velocity is difficult to determine by experiments.

The line-imaging velocity interferometer system for any reflector (VISAR) measures velocities by the phase change caused by Doppler-shifted light reflecting off a moving surface, using a probe laser that propagates through transparent sample [38]. It is sensitive to the shock front planarity, as it is usually used in laser dynamic shock compression experiments $[39,40]$. when shock front bend angle exceeds a certain threshold, the VISAR fringe signals are lost because probe beam can not go back into VISAR system. It is about $2^{\circ}-3^{\circ}$, depending on the VISAR system $f$ value (focus distance-diameter) of lenses. Thus, a method for detecting the speed of sound by lateral unloading wave was proposed by Li et al. in transparent materals [35] . The temporal lateral release trace in X-Y space and shock velocity $u_{s}$ are measured simultaneously by VISAR. When we assume that the threshold keeps q certain value when shock travels in time, the bend angle $\tan \alpha=X(t) / Y(t)$ can be obtained, where $Y(t)$ is the shock position, and $X(t)$ is edge rarefaction trace point position.

The advantage for determining speed of sound using the lateral release method is that it provides an absolute measurement for sound speed and the measurement only 
depends on shock velocity of shock front, spatial resolution of VISAR, and known Hugoniot $u_{s}-u_{p}$ relation.

However, it does not depend on properties from the reference material, which are required by the method from the extension to unsteady wave correction derived by Fratanduono et al. [41-43].

\subsection{Experimental Configuration and Targets}

The laser shock experiment was carried out at the Shenguang-III prototype laser facility (SG-IIIP), located at the Laser Fusion Research Center (CAEP) in Mianyang, China $[44,45]$. As a frequency-tripled Nd: glass laser, SG-IIIp facility operates at wave length of $351 \mathrm{~nm}$, which usually has 8 beams with total $1200 \mathrm{~J}$ for each with 1-3 nanosecond (ns) phase shaped pulse ability, and 9th beam as $4000 \mathrm{~J}$ in $5 \mathrm{~ns}$ with $20 \mathrm{~ns}$ pulse shaping.

In this experiment, the $\alpha$-quartz samples were shock compressed from X-ray indirectdrive in planar geometry. Up to 8 beams, delivering up to $800 \mathrm{~J}$ per beam of $351 \mathrm{~nm}$ UV laser in a 1-3 ns flat-top temporally shaped pulse, were focused on the $850 \mu \mathrm{m}$ laser entrance hole (LEH) of a $30 \mu \mathrm{m}$ thick, $3.6 \mathrm{~mm}$ length and $1.8 \mathrm{~mm}$ diameter gold hohlruam with $1 \mathrm{~mm} \times 0.5 \mathrm{~mm}$ diagnostic hole attaching with the target packages, as shown in Figure 1a. Distributed phase plates $500 \mu \mathrm{m}$ were used in this experiment to spatially smooth the beam profile and produce a super Gaussian intensity when projected onto the hohlruam wall. The uniform radiation field, with intense X-ray through absorption, was created in the gold hohlraum, which can deposit energy into target packages. The peak X-ray radiation temperature measured is $140-162 \mathrm{eV}$ in 1-2 ns, according to the maximum pressure needed to achieve in shots. Figure1b shows the typical X-ray radiation history in the hohlraum measured by the flat response X-ray diodes (FXRD) at $45^{\circ}$ angle in our experiment [46], in which peak temperature achieved up to $160 \mathrm{eV}$ at $1 \mathrm{~ns}$ in Shot 341 , and $145 \mathrm{eV}$ at $2 \mathrm{~ns}$ time in Shot 343.
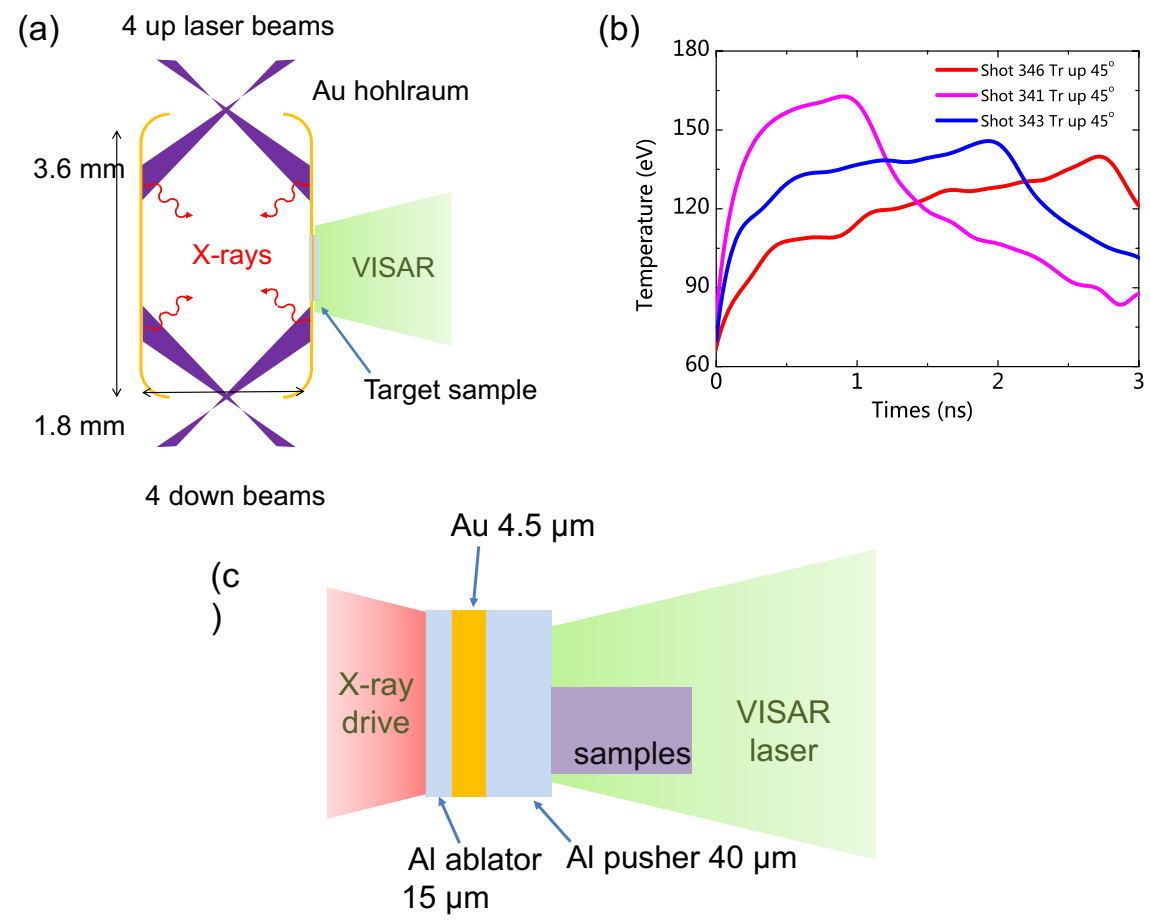

Figure 1. (a) Experimental setup of hohlraum-type with laser beams drive planar shock wave through X-ray. Line-imaging VISAR measures shock velocity and lateral release signal from target periphery. (b) Typical radiation profile of hohlraum temperature in shots from diagnostic device up $45^{\circ}$. (c) Target configuration in experiment with Al ablator, Au shield, Al pusher, and quartz sample. Free side in quartz sample was arranged in center of diagnostic hole, which is also perpendicular to Al pusher plane. 
Our target packages were $1.2 \mathrm{~mm} \times 1 \mathrm{~mm}$ flat and was consisted of a $15 \mu \mathrm{m}$ aluminum ablator for generating shock wave by $X$-ray ablation, a $4.5 \mu \mathrm{m}$ gold layer as $X$-ray preheat shield, and a further $35 \mu \mathrm{m}$ aluminum layer pusher to minimize impedance mismatch with sample and the $\alpha$-quartz baseplates, as shown in Figure 1c. For these experiments, 1-D hydro-dynamic simulations using the hydrocode MULTI-1D program[47] guided the peak shock pressure and temperature profile while shock propagates in target. Simulations and previous experiments also displayed negligible preheat after Al pusher for the shots at $180 \mathrm{eV}$ radiation temperature in $1.5 \mathrm{~ns}$. This confirms our sample was not preheated. The shock planar area from Al pusher is more than $500 \mu \mathrm{m}$ from previous experiments results [48-51]. Adjacent $\alpha$-quartz samples were glued onto the back of Al pusher layer using an $\mathrm{Sn}$ thin film as epoxy glue layers $<1 \mu \mathrm{m}$ thick. The free side of $\alpha$-quartz sample is perpendicular to the $\mathrm{Al}$ pusher plane and it is also located in the middle of $\mathrm{Al}$ pusher to make sure the bent shock front is only due to the edge rarefaction from free side of sample. The $\alpha$-quartz sample were $>250 \mu \mathrm{m}$ thick, with initial densities of $2.65 \mathrm{~g} / \mathrm{cm}^{3}$ and refractive index of 1.574 at $532 \mathrm{~nm}$.

\subsection{Experimental Diagnostic and Analysis}

Shock velocity and lateral release in quartz were measured using a line imaging VISAR system developed in Laser Fusion Research Center (LFRC). In this experiment, the pressure in quartz is above $200 \mathrm{GPa}$, where quartz melts and forms a reflecting front [15]. The raw VISAR images, as per the figure in Figure 2, were analyzed using the Fourier transform method to determine the phase [39]. To account for $2 \pi$ ambiguities in phase, two VISARs with different velocity sensitivities were used. Their sensitivities used were 5.10 and $7.30 \mathrm{~km} / \mathrm{s} /$ fringe, which correspond to velocity sensitivities of 3.24 and $4.64 \mathrm{~km} / \mathrm{s} /$ fringe in quartz. The VISAR images provide phase shifts resulting from changes in velocity that were analyzed using the Fourier transform method [40]. Uncertainty in determining the phase was estimated as $3 \%$ of a fringe, which resulted in $<1 \%$ uncertainties due to multiple fringe jumps [40]. The VISAR system used a frequency-doubled Nd: YAG laser operating at $532 \mathrm{~nm}$, and the two streak cameras used $20 \mathrm{~ns}$ sweep durations, resulting in temporal sensitivities of $30 \mathrm{ps}$. A sample of an extracted shock velocity profile and edge rarefaction boundary where no signal returns from the shock front is given in Figure 2.

The shock velocity $u_{s}$ in the quartz was measured immediately after shock break out of Al pusher. A weak smoothing splice was applied to the velocities to mitigate the influence of random noise on the fitting routine. The first step is to select points of edge rarefaction wave trace when it incidentally enters into the shock from the side. The points were chosen within boundary of bending fringes with certain relatively intense clim. The horizontal pixels difference $d X$ and vertical time difference $d t$ between initial and final points in VISAR images infers the edge rarefaction wave traveling distance $X$ and position of the shock front $Y(t)$, as $X=M \times d X$ and $Y(t)=\int_{t_{0}}^{t_{0}+d t} u_{s}(t) d t$, where $t_{0}$ is the time for starting point. The parameter $M$ is the spatial resolution factor for VISAR, which is measured in static experiment before dynamical experiments. The spatial scaling $M_{L}=0.536[\mu \mathrm{m} /$ pixel $]$ and $M_{R}=0.64[\mu \mathrm{m} /$ pixel $]$ with certain uncertainty respectively for VISAR $L$ and R legs in this experiment. The angle $\alpha$ can be obtained from the $X(t)$ and $Y(t)$ profile as $\tan \alpha=X(t) / Y(t)$.

In this work, the quartz Hugoniot data and the $u_{s}-u_{p}$ relation for $\alpha$-quartz is used as $u_{s}=a+b u_{p}+c u_{p}^{2}+d u_{p}^{3}$, where $a=6.278, b=1.193, c=2.505, d=0.3701$. The particle velocity $u_{p}$ is solved by measurement of shock velocity $u_{s}$ from VISAR with uncertainty considering covariance matrix elements for $u_{s}-u_{p}$ relation by Knudson and Desjarlais [27]. We found that sound speed profile is highly dependent on the points from bent trace in VISAR images and spatial resolution of VISAR system. In our improved analysis, the average sound speed for each shot was obtained using average shock velocity between initial and final bend points, as shock density changes a little when it decays in the indirect drive experiments. This average speed of sound analysis method could mitigate the 
misleading information on sound speed coming from the bend trace points selecting spatial resolution $4-5 \mu \mathrm{m}$ and random noise in VISAR images.

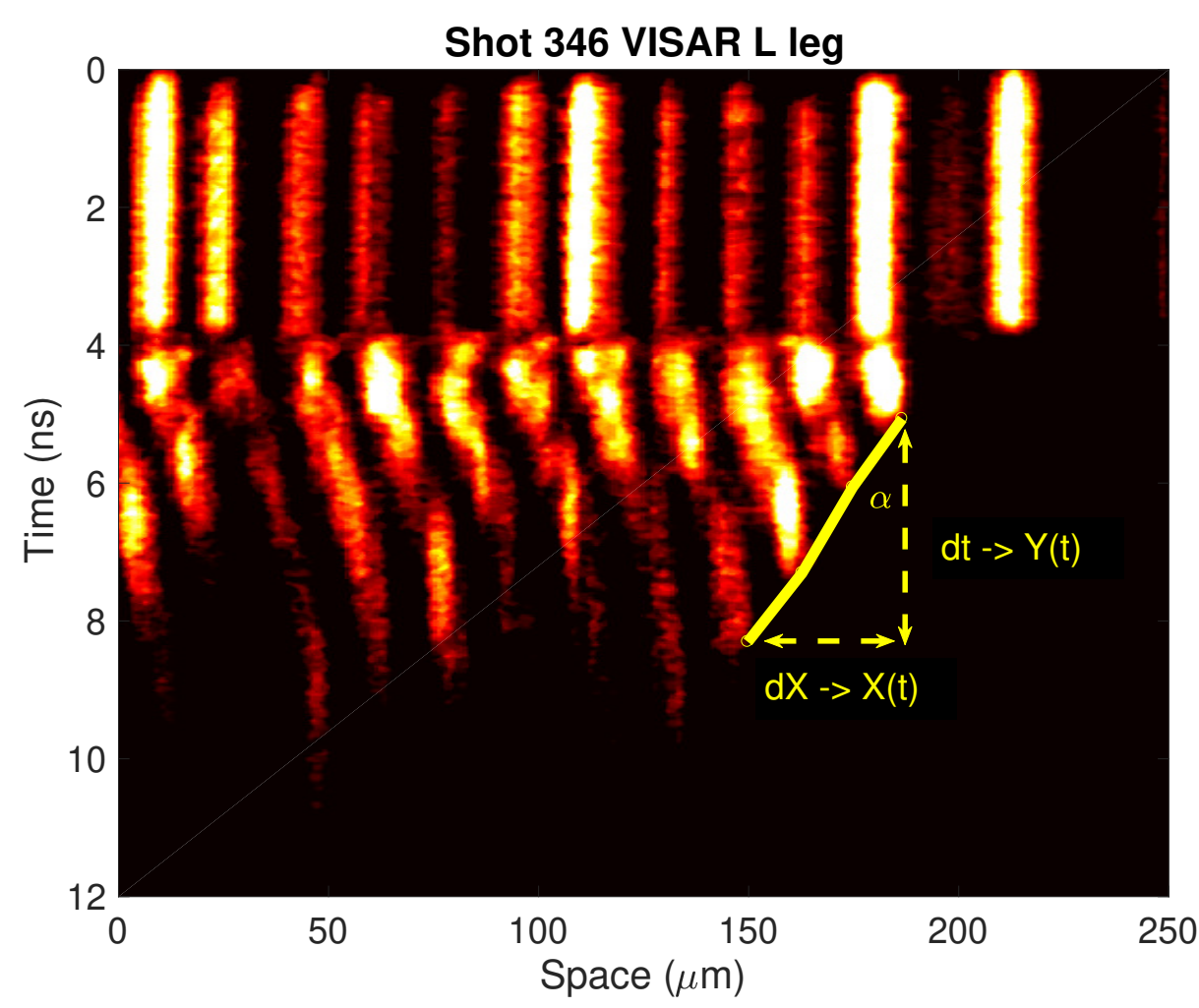

Figure 2. Raw VISAR data for shot 346. Line-imaging VISAR record provides 1-D spatial information of the shock velocity as a function of time while shock propagates through quartz sample. The edge rarefaction from free side interacts with shock wave to bend the shock wave front. The yellow line in this figure presents the trace of edge rarefaction in sample, which could be infer sound velocity with shock velocity measured from the moving fringes in VISAR images.

Uncertainties were calculated using the Monte Carlo method, which generates normally distributed random variables based on the mean and standard deviation of an observed quantity. In these experiments, independent variables in the Monte Carlo simulations were: the measured shock velocities in the quartz, coefficients in quartz Hugoniot $u_{s}-u_{p}$ relation, spatial scaling parameter M for VISAR system, spatial and temporal resolution of streak cameras. The variances in the initial density of the $\alpha$-quartz is assumed to be negligible. The uncertainty in the measured velocities and coefficients in Hugoniot relation were used to determine the corresponding uncertainty in pressure, density, and particle velocity. The measured sound velocity was determined from the average of the Monte Carlo calculations for each shot. The system errors such as M parameter, VISAR spatial resolution mainly resulted in uncertainty $4-8 \%$ of sound velocities, while the random errors was estimated to be less than $1 \%$. This level of total uncertainty is similar to techniques using laser unsteady wave method [42,43] and overtaking method with gas guns [32,33,52]. But this lateral release method provide an absolutely determined sound velocity and the uncertainties from unsteady wave method may be underestimated because they do not consider the uncertainties coming from sound velocity standard [42,43].

\section{Results}

The speed of sound for shocked liquid quartz was measured at pressure from 270 to $900 \mathrm{GPa}$ in 7 shots experiment, as listed in Table 1. The edge rarefaction wave traces in VISAR images could generate a series of well-constrained data. The equation of state parameters for shock compressed quartz was found by the shock velocity and Hugoniot 
relation [27]. As mentioned previously, the speed of sound was calculated using the average velocity and the average edge rarefaction angle over the region. The uncertainty in the measurement of the sound velocity ranges from $4 \%$ to $10 \%$.

Table 1. Experimental results on shock velocity $\left(u_{s}\right)$, pressure $(\mathrm{P})$, density $(\rho)$, sound velocity $\left(C_{B}\right)$, and Grüneisen parameter $(\gamma)$ of shocked quartz.

\begin{tabular}{|c|c|c|c|c|c|}
\hline Shot No. & $u_{s}(\mathrm{~km} / \mathrm{s})$ & P (GPa) & $\rho(\mathrm{g} / \mathrm{cc})$ & $C_{B}(\mathrm{~km} / \mathrm{s})$ & $\gamma$ Parameter \\
\hline 340 & $21.63 \pm 0.16$ & $742.68 \pm 11.91$ & $6.71 \pm 0.16$ & $16.69 \pm 0.66$ & $0.76 \pm 0.06$ \\
\hline 341 & $23.18 \pm 0.16$ & $871.28 \pm 18.55$ & $6.91 \pm 0.20$ & $15.30 \pm 0.97$ & $0.87 \pm 0.07$ \\
\hline 342 & $19.66 \pm 0.23$ & $605.36 \pm 8.12$ & $6.43 \pm 0.12$ & $13.88 \pm 0.54$ & $0.99 \pm 0.05$ \\
\hline 343 & $16.86 \pm 0.16$ & $430.51 \pm 13.94$ & $6.05 \pm 0.06$ & $13.94 \pm 0.51$ & $0.97 \pm 0.06$ \\
\hline 344 & $16.68 \pm 0.16$ & $412.42 \pm 4.04$ & $6.03 \pm 0.06$ & $13.06 \pm 0.51$ & $1.07 \pm 0.06$ \\
\hline 345 & $16.33 \pm 0.16$ & $391.78 \pm 3.73$ & $5.98 \pm 0.05$ & $14.14 \pm 0.90$ & $0.94 \pm 0.11$ \\
\hline 346 & $13.69 \pm 0.16$ & $268.36 \pm 2.29$ & $5.64 \pm 0.03$ & $12.95 \pm 1.34$ & $1.00 \pm 0.20$ \\
\hline
\end{tabular}

The data for sound velocity in silica at high pressure are summarized in Figure 3. As shown in this figure, the black squares [34] and triangles [31] represent longitudinal sound velocity data measured for crystal quartz, respectively, along quartz shock Hugoniot and spanning shock melting pressure around $200 \mathrm{GPa}$.

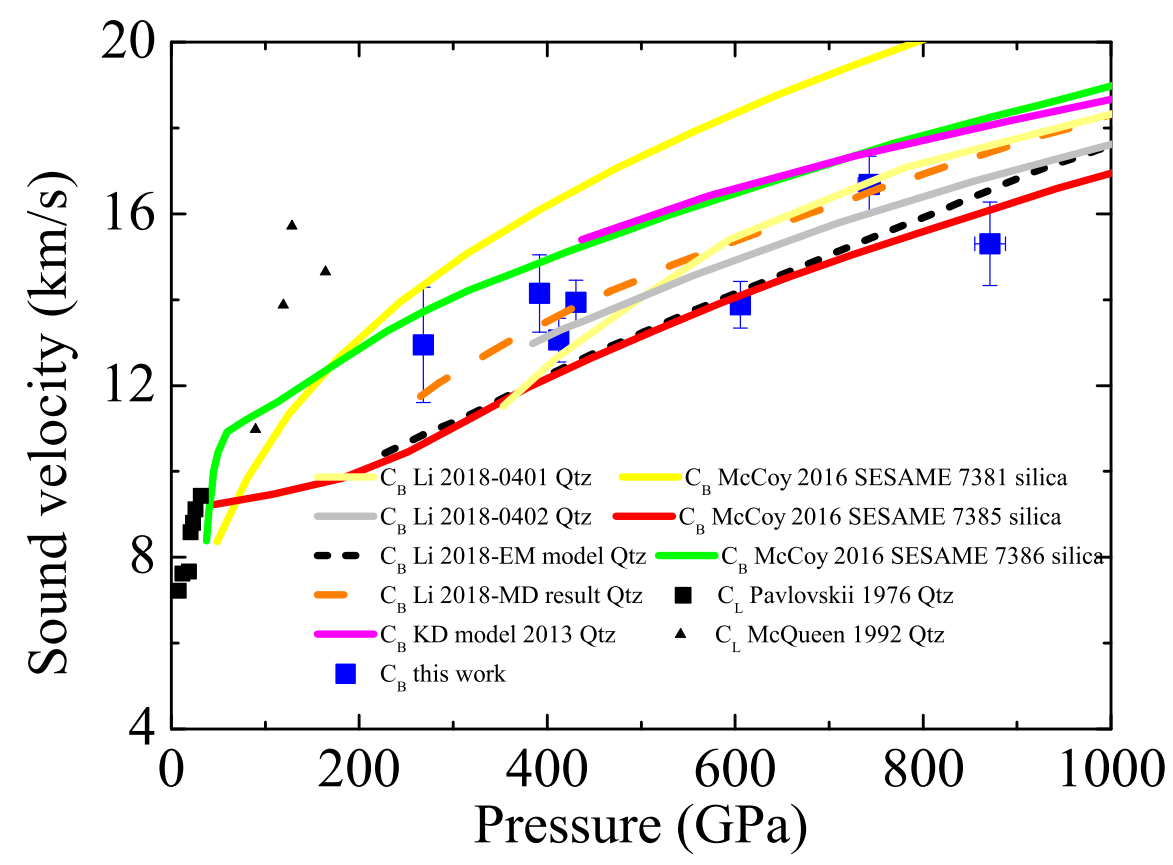

Figure 3. Measured isentropic sound velocity (blue squares with error bars) is in good agreement with 2 reported shots (light grey and yellow line) and DFT-MD calculations (orange line) in Ref. [35], as well as previous data from Pavlovskii (black squares) and McQueen results (black triangles) at pressure below $500 \mathrm{GPa}$. At higher pressure to $900 \mathrm{GPa}$, our data prefer SESAME 7385 for silica (red line) in Ref. [42] and empirical EOS model (EM model) results for quartz (black dashed line). In this figure, $C_{b}$ means bulk sound velocity, $C_{L}$ means longitudinal sound velocity, and $Q t z$ means quartz sample.

The sound speed data from theoretical models for quartz were also plotted in Figure 3, which were calculated for initial density of fused silica as summarized in Ref. [42]. In this figure, theoretical model results from the analytic solutions (KD model) from the release isentrope [27], density functional theory molecular dynamics (DFT-MD) calculations and empirical model (EM model) from wide regime equation of state (WEOS) [35] were also presented. Most of our measured sound velocities with errors are located in the region 
where theoretical model data results cover the whole pressure regions from $200 \mathrm{GPa}$ to $1000 \mathrm{GPa}$, but slightly lower than those data from KD model, SESAME EOS table 7381 and 7386 for silica [42]. The present sound speed results could be analyzed in two parts. At lower pressure of $200 \mathrm{GPa}$ to $500 \mathrm{GPa}$, the sound velocity agrees with decay-laser shock results and DFT-MD results in Ref. [35]. But as the pressure goes above $600 \mathrm{GPa}$, our sound velocity data indicate a gentle increase in slope at high pressures from the whole data, compared with the previous theoretical and experimental results.

Liquid silica at extremely high shock compression undergo a significant dissociation transition where $\mathrm{SiO}_{2}$ dissociates atomic $\mathrm{Si}$ and $\mathrm{O}$. The broad peak in specific heat and $u_{s}-u_{p}$ Hugoniot curve observed in experiments were determined for indicating shortrange structure or the energetics of bond breaking [24,27]. The bulk sound velocity behavior in 700-900 GPa may infer this bonded to atom liquid phase transition in quartz. A smooth increase in present sound velocity data is shown in this figure, although some are beyond the errors, and more experimental data for shocked quartz around pressure 700-850 GPa are still lacking, which could help to show more clear changes without being misled by the experimental uncertainties of data.

The Grüneisen parameter $\gamma$, defined by $\gamma=V\left(\frac{d P}{d E}\right)_{V}$, is a fundamental thermodynamic quantity with wide applications. For liquid silica, it affects the adiabatic temperature gradient and the amount of heat conducted along the adiabatic. It was assumed to be a constant by different model by DFT-MD calculations [27]. $\gamma$ can be determined from the slope of Hugoniot data and the isentrope at a point in phase space, which is related as

$$
\gamma=\frac{2\left(V\left(\frac{d P}{d V}\right)_{H}+\frac{C_{s}^{2}}{V}\right)}{P_{H}+\left(\frac{d P}{d V}\right)_{H}\left(V_{0}-V_{H}\right)}
$$

where $(d P / d \rho)_{H}$ is the slope of Hugoniot curve at volume $V_{H}$ and pressure $P$, subscript 0 indicates zero-pressure conditions and $C_{S}$ is the isentropic sound velocity at Hugoniot state.

In this work, $\gamma$ parameter for liquid quartz at high pressure was calculated using Equation (2) for each shot. The uncertainty in Grüneisen parameter was also calculated using the Monte Carlo method to be 5\% to 20\%, which depends on the pressure. The slope $(d P / d \rho)_{H}$ is adopted by Rankine-Hugoniot relationship in Ref. [27]. The increasing uncertainty in the slope of Hugoniot at high pressures also increases that in $\gamma$ parameter.

Figure 4 presents our data and previous data from experimental and theoretical models. Most of results at higher pressure fall into the region bounded by $V \Delta P / \Delta E$ average values (light blue line) and DFT-MD results (blue line) in Ref. [35]. The Grüneisen parameter decreases with shock temperature and pressure over the experimental range in general view. But they also show a slower slope comparing with that of experimental results (0401 black line and 0402 red line) and DFT-MD calculations. They also do not favor any SESAME table, which predicted a significantly stiffer result. For the densities above $6.0 \mathrm{~g} / \mathrm{cm}^{3}$, experimental data approach $0.7-0.8$. This is in agreement with previous results that implied the validity of a constant $\gamma$ parameter for liquid silica above high pressure $400 \mathrm{GPa}$. But this value is bigger compared with the constant (the value for the ideal gas.) $\gamma=0.66$ (black dashed line) in Refs. [27,42]. The data in this work also show a slower decay in $\gamma$ parameter, which is directly relevant to the sound velocity behavior at high pressure. Although more data are still needed to make up around the regions in future experiments, they may infer the boned-atomic dissociation phase transition in liquid quartz, as $\gamma$ parameter directly relates to the pressure and energy, which is required to break chemical bonds for dissociation of liquid $\mathrm{SiO}_{2}$ molecule into $\mathrm{Si}$ and $\mathrm{O}$ atom. 


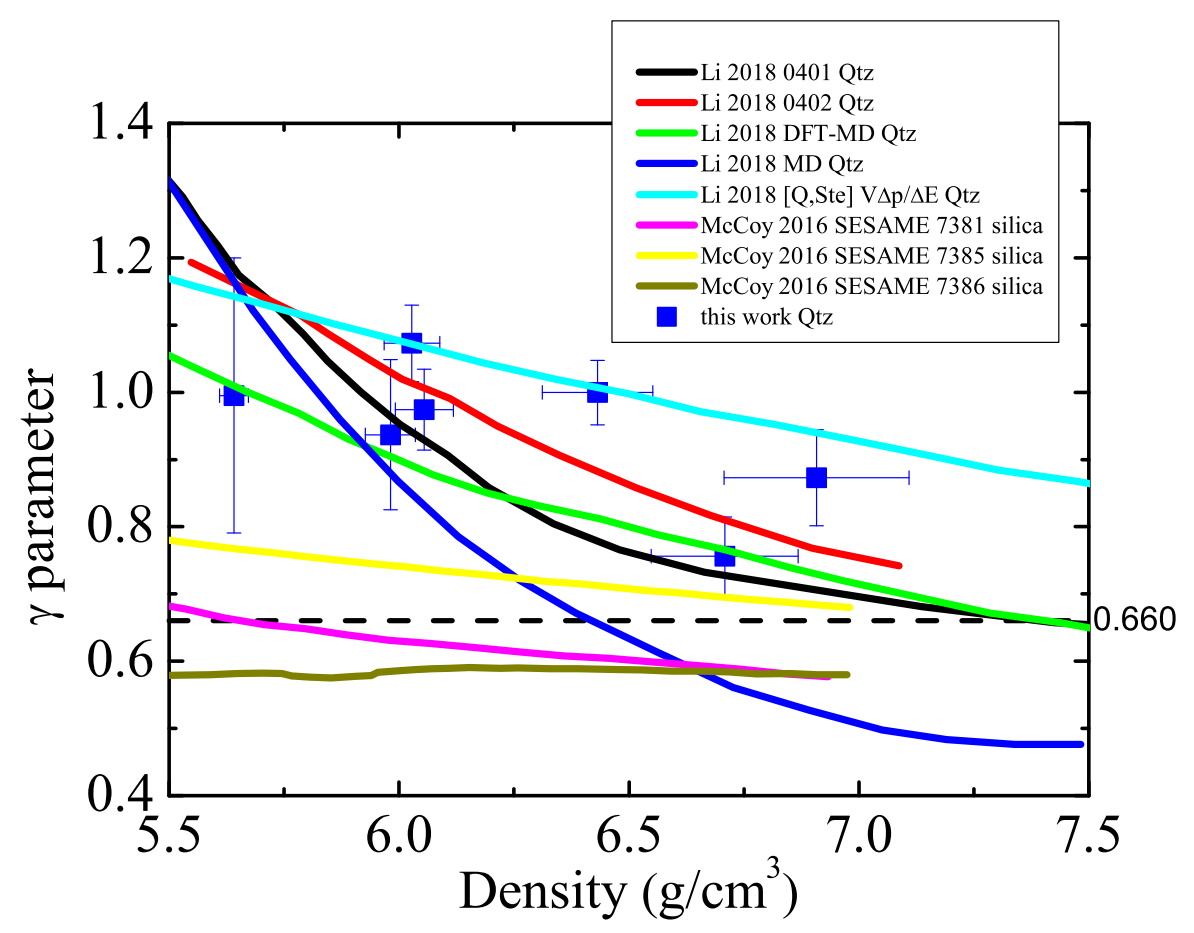

Figure 4. Measurements of Grüneisen parameter in quartz (blue square with error bars). Most of present results fall into region bounded by average values (light blue line) and DFT-MD results (blue line) in Ref. [35]. All data do not favor any SESAME table Ref. [42] and are greater than the theoretical limit $\gamma=0.66$. The Grüneisen parameter measured is in agreement with previous experimental data in 0401 (black line) and 0402 (blue line), as density is below $6.2 \mathrm{~g} / \mathrm{cm}^{3}$. The slower decay process in the Grüneisen parameter was found with consideration of the errors as the density goes from 6.5 to $7.0 \mathrm{~g} / \mathrm{cm}^{3}$.

\section{Conclusions}

The sound velocity of shocked $\alpha$-quartz was measured using lateral release wave from the periphery of the crystal in the regions of 250 to $900 \mathrm{GPa}$, where it is melted. The improved analysis method in the present study demonstrates lateral release method for directly measuring the sound velocity by VISAR, proposed by Li et al. [35], when Rankine-Hugoniot relation is known. The present results are consistent with previous experimental results and predictions by DFT-MD. The sound velocity in liquid quartz above 600 GPa shows more gentle slope than previous experimental results and prefers SEASAME EOS 7385 for silica [42] and the empirical model [35]. The uncertainties by Monte Carlo calculations are similar to those of the previous results with uncertainties. The Grüneisen parameter was also calculated from sound velocity and quartz Hugoniot data, and the result decreases with increasing shock pressure. The results agree with the theoretical models as a shallow slope relative to the pressure. The behavior for measured sound velocity and $\gamma$ parameter at extreme high pressures may be directly related to the boned to atomic phase transition found in shocked liquid silica at high pressure, although more experimental data should be provided in future experiments.

The sound speed revisited in our experiment provide further information on dissociation and metallization for liquid quartz at extreme conditions. The more gentle behavior we observed in present experiments may give us a new vision about transport properties of high-pressure liquid silica, although it requires further study. Our agreements with previous results increase our confidence in our theoretical model in Super-Earth interior conditions and help our understanding of the properties of magma oceans and melting on exoplanets. 
Author Contributions: Project lead, T.S., L.S.; experiment participants, W.Y., Y.Z., Z.G., Y.Z., X.D.; formal analysis, Y.Z.; investigation, L.S., Z.W.; resources, Z.W., J.Y.; data analysis, L.S., H.Z.; writingoriginal draft preparation, L.S. and H.Z.; writing, review, and editing, L.S., Y.Z. and T.S. All authors have read and agreed to the published version of the manuscript.

Funding: This work was supported by the National Key R\&D Program of China under Contract No. 2017YFA0403201 and the National Natural Science Foundation of China No. 12074351.

Data Availability Statement: All VISAR results and sample characterization data are available upon request.

Acknowledgments: The authors would like to thank the technical team members, Zhi Jun Li, Sai Jin, Run Chang Zhao, Meng Shen Yang, Tai Ming Yi, and Wei Chao Tong at LFRC, for their invaluable work producing the targets and operating the facility. Liang Sun would like to thank Shao Yong Tu at LFRC for helpful discussion on data analysis. The authors also want to thank Hua Shu for helpful discussion on physical analysis.

Conflicts of Interest: The authors declare no conflict of interest.

\section{References}

1. Duffy, T.; Madhusudhan, N.; Lee, K.K.M. Mineralogy of Super-Earth Planets. In Treatise on Geophysics; Schubert, G., Ed.; Elsevier: Amsterdam, The Netherlands, 2015; pp. 393-416.

2. Rauer, H.; Catala, C.; Aerts, C.; Appourchaux, T.; Benz, W.; Brandeker, A.; Christensen-Dalsgaard, J.; Deleuil, M.; Gizon, L.; Goupil, J.; et al. The PLATO 2.0 mission. Exp. Astron. 2014, 38, 249-330. [CrossRef]

3. Lissauer, J.; Dawson, R.I.; Tremaine, S. Advances in exoplanet science from Kepler. Nature 2014, 513, 336-344. [CrossRef]

4. Valencia, D.; Connel, R.J.O.; Sasselov, D. Internal structure of massive terrestrial planets. Icarus 2006, 181, 545-554. [CrossRef]

5. Swift, D.C.; Eggert, J.H.; Hicks, D.C.; Hamel, S.; Caspersen, K.; Schwegler, E.; Collins, G.W.; Nettelmann, N.; Ackland, G.J. Mass-radius relationships for exoplanets. Astrophys. J. 2012, 744, 59. [CrossRef]

6. Zeng, L.; Sasselov, D.D.; Jacobsen, S.B. Mass-radius relation for rocky planets based on Prem. Astrophys. J. 2016, 819, 127. [CrossRef]

7. Unterbornal, C.T.; Dismukes, E.E.; Panero, W.R. Scaling the Earth: A sensitivity analysis of terrestrial exoplanetary interior models. Astrophys. J. 2016, 819, 32. [CrossRef]

8. Olson, P. Mantle control of the geodynamo: Consequences of top-down regulation. Geochem. Geophys. Geosyst. 2006, 17, 1935-1956. [CrossRef]

9. Lammer, H. What makes a planet habitable? Astron. Astrophys. Rev. 2009, 17, 181-249. [CrossRef]

10. Soubiran F.; Militzer B. Electrical conductivity and magnetic dynamos in magma oceans of Super-Earths. Nat. Comm. 2018, 9, 3883. [CrossRef]

11. Stixrude, L. Melting in Super-Earths. Philos. Trans. R. Soc. A 2014, 372, 20130076. [CrossRef]

12. Hemley, R.; Prewitt, C.T.; Kingma, K.J. High-pressure behavior of silica. In Silica: Physical Behavior, Geochemistry and Materials Applications; Manghnani, P.J., Prewitt, C.T., Gibbs, G.V., Eds.; Mineralogical Society of America: Blacksburg, Virginia, 1994; Volume 29, pp. 41-81.

13. Wentzcovitch, R.M.; Silva, C.D.; Chelikowsky, J.R.; Binggeli, N. A new phase and pressure induced amorphization in silica. Phys. Rev. Lett. 1998, 80, 2149-2152. [CrossRef]

14. Haines, J.; Leger, J.M.; Gorelli, F.; Hanfland, M. Crystalline post-quartz phase in silica at high pressure. Phys. Rev. Lett. 2001, 87, 155503. [CrossRef] [PubMed]

15. Akins, J.A.; Ahrens, T.J. Dynamic compression of $\mathrm{SiO}_{2}$ : A new interpretation. Geophys. Res. Lett. 2002, 29, 31-1-31-4. [CrossRef]

16. Luo, S.-N.; Akins, J.A.; Ahrens, T.J.; Asimov, P.D. Shock-compressed $\mathrm{MgSiO}_{3}$ glass, enstatite, olivine, and quartz: Optical emission, temperatures, and melting. J. Geophys. Rev. 2004, 109, B05205. [CrossRef]

17. Dubrovinsky, L.S.; Dubrovinskaia, N.A.; Prakapenka, V.; Seifert, F.; Langenhorst, F.; Dmitriev, V.; Weber, H.-P.; Bihan, T.L. A class of new high-pressure silica polymorphs. Phys. Earth Planet. Int. 2004, 143-144, 231-240. [CrossRef]

18. Hu, Q.Y.; Shu, J.-F.; Yang, W. G.; Park, C.; Chen, M. W.; Fujita, T.; Mao, H.-K.; Sheng, H. W. Stability limits and transformation pathways of $\alpha$-quartz under high pressure. Phys. Rev. B 2017, 95, 104112. [CrossRef]

19. Tracy, S.J.; Turneaure, S.J.; Duffy, T.S. In situ X-Ray Diffraction of Shock-Compressed Fused Silica. Phys. Rev. Lett. 2018, 120, 137702. [CrossRef]

20. Prescher, C.J.; Prakapenka, V.B.; Stefanski, J.; Jahn, S.; Skinner, L.B.; Wang, Y.-B. Beyond sixfold coordinated $\mathrm{Si}_{\text {in }} \mathrm{SiO}_{2}$ glass at ultrahigh pressures. Proc. Natl. Acad. Sci. USA 2017, 114, 10041-10046. [CrossRef] [PubMed]

21. Asimov, P.D. Dynamic compression. In Treatise on Geophysics; Schubert, G., Ed.; Elsevier: Amsterdam, The Netherlands, 2015; pp. 393-416.

22. Hicks, D.G.; Boehly, T.R.; Celliers, P.M.; Eggert, J.H.; Vianello, E.; Meyerhofer, D.D.; Collins, G.W. Shock compression of quartz in the high-pressure fluid regime. Phys. Plasmas 2005, 12, 082702. [CrossRef] 
23. Knudson, M.D.; Desjarlais, M.P. Shock Compression of Quartz to 1.6 TPa: Redefining a Pressure Standard. Phys. Rev. Lett. 2009, 103, 225501. [CrossRef]

24. Hicks, D.G.; Beohly, T.R.; Eggert, J.H.; Miller, J.E.; Celliers, P.M.; Collins, G.W. Dissociation of Liquid Silica at High Pressures and Temperatures. Phys. Rev. Lett. 2006, 97, 025502. [CrossRef] [PubMed]

25. Qi, T.; Millot, M.; Kraus, R.G.; Root, S.; Hamel, S. Optical and transport properties of dense liquid silica. Phys. Plasmas 2015, 22, 062706. [CrossRef]

26. Boehly, T.R.; Miller, J.E.; Meyerhofer, D.D.; Eggert, J.H.; Celliers, P.M.; Hicks, D.G.; Collins, G.W. Shock Compression of Conducted Matter-2007; Elert, M., Furnish, M.D., Chau, R., Holmes, N., Nguyen, J., Eds.; American Institute of Physics: Melville, NY, USA, 2007; Volume 955, p. 19.

27. Knudson, M.D.; Desjarlais, M.P. Adiabatic release measurements in $\alpha$-quartz between 300 and 1200 GPa: Characterization of $\alpha$-quartz as a shock standard in the multimegabar regime. Phys. Rev. B 2013,88, 184107. [CrossRef]

28. Kraus, R.G.; Stewart, S.T.; Swift, D.C.; Bolme, C.A.; Smith, R.F.; Hamel, S.; Hammel, B.D.; Spaulding, D.K.; Hicks, D.G.; Eggert, J.H.; et al. Shock vaporization of silica and the thermodynamics of planetary impact events: Shock vaporization of silica. J. Geophys. Res. 2012, 117, E09009. [CrossRef]

29. Millot, M.; Dubrovinskaia, N.; Cernok, A.; Blaha, S.; Dubrovinsky, L.; Braun, D.G.; Celliers, P.M.; Collins, G.W.; Eggert, J. H.; Jeanloz, R. Shock compression of stishovite and melting of silica at planetary interior conditions. Science 2015, 347, 418 [CrossRef]

30. Denoeudn, A.; Benuzzi-Mounaix, A.; Ravasio, A.; Dorchies, F.; Leguay, P.M.; Gaudin, J.; Guyot, F.; Brambrink, E.; Koenig, M.; Le Pape, S.; et al. Metallization of Warm Dense $\mathrm{SiO}_{2}$ Studied by XANES Spectroscopy. Phys. Rev. Lett. 2014, 113, 116404. [CrossRef]

31. Pavlovskii, M.N. Measurements of the velocity of sound in shock-compressed quartzite, dolomite, anhydrite, sodium chloride, paraffin, plexiglas, polyethylene, and fluoroplast-4. J. Appl. Mech. Tech. Phys. 1976, 17, 709. [CrossRef]

32. Duffy, T.S.; Ahrens, T.J. Sound velocities at High pressure and Temperature and Their Geophysical Implications. J. Geophys. Res. 1992, 97, 4503-4520. [CrossRef]

33. Tan, Y.; Yu, Y.Y.; Dai, C.-D.; Jin, K.; Wang, Q.S.; Hu, J.-B.; Tan, H. Hugoniot and sound velocity measurements of bismuth in the range of 11-70 GPa. J. Appl. Phys. 2013, 113, 093509. [CrossRef]

34. McQueen, R.G. The Velocity of Sound behind Strong Shocks in $\mathrm{SiO}_{2}$. In Shock Compression of Condensed Matter-1991; Dick, R.D., Forbes, J.W., Tasker, D.G., Eds.; Elsevier: Amsterdam, The Netherlands, 1992; p. 75.

35. Li, M.; Zhang, S.; Zhang, H.P.; Zhang, G.M.; Wang, F.; Zhao, J.H.; Sun, C.W.; Jeanloz, R. Continuous Sound Velocity Measurements along the Shock Hugoniot Curve of Quartz. Phys. Rev. Lett. 2018, 120, 215703. [CrossRef]

36. Altshuler, V.L.; Kormer, S.B.; Brazhnik, M.I.; Vladimirov, L.A.; Speranskaya, M.P.; Funtikov, A.I. The isentropic compression of aluminum, copper, lead and iron at high pressures. Sov. Phys. JETP 1960, 11, 766.

37. Zeldovich, Y.B.; Raizer, Y.P. Physics of Shock Waves and High Temperature Hydrodynamic Phenomena; Hayes, W.D., Probstein, R.F., Eds.; Dover Publications, Inc.: Mineola, NY, USA, 2002.

38. Barker, L.M.; Hollenbach, R.E. Laser interferometer for measuring high velocities of any reflecting surface. J. Appl. Phys.1972, 43, 4669. [CrossRef]

39. Celliers, P.M.; Collins, G.W.; Da Silva, L.B.; Gold, D.M.; Cauble, R. Accurate measurement of laser-driven shock trajectories with velocity interferometry. Appl. Phys. Lett. 1998, 73, 1320. [CrossRef]

40. Celliers, P.M.; Bradley, D.K.; Collins, G.W.; Hicks, D.G.; Boehly, T.R.; Armstrong, W.J. Line-imaging velocimeter for shock diagnostics at the OMEGA laser facility. Rev. Sci. Instrum. 2004, 75, 4916. [CrossRef]

41. Fratanduono, D.E.; Munro, D.H.; Celliers, P.M.; Collins, G.W. Hugoniot experiments with unsteady waves. J. Appl. Phys. 2014, 116, 033517. [CrossRef]

42. McCoy, C.A.; Gregor, M.C.; Polsin, M.C.; Fratanduono, D.E.; Celliers, P.M.; Boehly, T.R.; Meyerhofer, D.D. Measures of the sound velocity of shock-compressed liquid silica to $1100 \mathrm{GPa}$. J. Appl. Phys. 2016, 120, 235901. [CrossRef]

43. McCoy, C.A.; Hu, S.X.; Marshall, M.C.; Polsin, M.C.; Fratanduono, D.E.; Ding, Y.H. Celliers, P.M.; Boehly, T.R.; Meyerhofer, D.D. Measurement of the sound velocity and Grüneisen parameter of polystyrene at inertial confinement fusion conditions. Phys. Rev. B 2020, 102, 184102. [CrossRef]

44. Zheng, W.-G.; Wei, X.-F.; Zhu, Q.-H.; Jing, F.; Hu, D.-X.; Su, J.-Q.; Zheng, K.-X.; Yuan, X.-D.; Zhou, H.; Dai, W.-J.; et al. Laser performance of the SG-III laser facility. High Power Laser Sci. Eng. 2016, 4, e21. [CrossRef]

45. Gong, T.; Hao, L.; Li, Z.-C.; Yang, D.; Li, S.-W.; Guo, L.; Zou, S.-Y.; Liu, Y.-Y.; Jiang, X.-H.; Peng, X.-S.; et al. Recent research progress of laser plasma interactions in Shenguang laser facilities. Matter Radiat. Extremes. 2019, 4, 055202. [CrossRef]

46. Li, Z.-C.; Jiang, X.-H.; Liu, S.-Y.; Huang, T.-X.; Zheng, J.; Yang, J.-M.; Li, S.-W.; Guo, L.; Zhao, X.-F.; Du, H.-B.; et al. A novel flat-response X-ray detector in the photon energy range of 0.1-4 keV. Rev. Sci. Instrum. 2010, 81, 073504. [CrossRef]

47. Ramis, R.; Meyer-ter-Vehn, J. MULTI-IFE-A one-dimensional computer code for Inertial Fusion Energy (IFE) target simulations, Comput. Phys. Commun. 2016, 203, 226-237. [CrossRef]

48. Liu, W.; Duan, X.-X.; Jiang, S.-E.; Wang, Z.-B.; Sun, L.; Liu, H.; Yang, W.-M.; Zhang, H.; Ye, Q.; Li, Y.-Y.; et al. Laser-driven shock compression of gold foam in the terapascal pressure range. Phys. Plasmas. 2018, 25, 062707. [CrossRef]

49. Hartley, N. J.; Zhang, C.; Duan, X.-X.; Huang, L.-G.; Jiang, S.-E.; Li, Y.; Yang, L.; Pelka, A.; Wang, Z.-B.; Yang, J.-M.; et al. Dynamically pre-compressed hydrocarbons studied by self-impedance mismatch. Matter Radiat. Extrem. 2020, 5, 028401. [CrossRef] 
50. Duan, X.-X.; Zhang, C.; Guan, Z.-Y.; Sun, L.; Peng, X.-S.; Liu, H.; Yang, W.-M.; Li, Y.-L.; Zhang, H.; Ye, Q.; et al. Transparency measurement of lithium fluoride under laser-driven accelerating shock loading. J. Appl. Phys. 2020, 128, 015902. [CrossRef]

51. Wang, P.; Zhang, C.; Jiang, S.-E.; Duan, X.-X.; Zhang, H.; Li, L.-L.; Yang, W.-M.; Liu, Y.-G.; Li, Y.-L.; Sun, L.; et al. Densitydependent shock Hugoniot of polycrystalline diamond at pressures relevant to ICF. Matter Radiat. Extrem. $2021,6,033902$. [CrossRef]

52. Berryman, E.J.; Winey, J.M.; Gupta, Y.M.; Duffy, T.S. Sound Velocities in Shock-Synthesized Stishovite to 72 GPa. Geophys. Res. Lett. 2019, 46, 13,697-13.703. [CrossRef] 